\title{
Effect of tungsten and molybdenum on growth of a syntrophic coculture of Syntrophobacter fumaroxidans and Methanospirillum hungatei
}

\author{
Caroline M. Plugge $\cdot$ Bo Jiang $\cdot$ Frank A. M. de Bok \\ Chingling Tsai $\cdot$ Alfons J. M. Stams
}

Received: 19 June 2008 / Revised: 15 August 2008 / Accepted: 21 August 2008 / Published online: 16 September 2008

(C) The Author(s) 2008. This article is published with open access at Springerlink.com

\begin{abstract}
The effect of tungsten (W) and molybdenum (Mo) on the growth of Syntrophobacter fumaroxidans and Methanospirillum hungatei was studied in syntrophic cultures and the pure cultures of both the organisms. Cells that were grown syntropically were separated by Percoll density centrifugation. Measurement of hydrogenase and formate dehydrogenase levels in cell extracts of syntrophically grown cells correlated with the methane formation rates in the co-cultures. The effect of $\mathrm{W}$ and Mo on the activity of formate dehydrogenase was considerable in both the organisms, whereas hydrogenase activity remained relatively constant. Depletion of tungsten and/or molybdenum, however, did not affect the growth of the pure culture of $S$. fumaroxidans on propionate plus fumarate significantly, although the specific activities of hydrogenase and especially formate dehydrogenase were influenced by the absence of Mo and W. This indicates that the organism has a low $\mathrm{W}$ or Mo requirement under these conditions. Growth of $M$. hungatei on either formate or $\mathrm{H}_{2} / \mathrm{CO}_{2}$ required tungsten, and molybdenum could replace tungsten to some extent. Our results suggest a more prominent role for $\mathrm{H}_{2}$ as electron carrier in the syntrophic conversion of propionate, when the essential trace metals $\mathrm{W}$ and Mo for the functioning of formate dehydrogenase are depleted.
\end{abstract}

Keywords Formate dehydrogenase $\cdot$ Hydrogenase . Interspecies hydrogen and formate transfer $\cdot$ Propionate . Tungsten $\cdot$ Molybdenum $\cdot$ Syntrophy

Communicated by Friedrich Widdel.

C. M. Plugge $(\square) \cdot$ B. Jiang $\cdot$ F. A. M. de Bok $\cdot$ C. Tsai ·

A. J. M. Stams

Laboratory of Microbiology, Wageningen University,

Dreijenplein 10, 6703 HB Wageningen, The Netherlands

e-mail: caroline.plugge@wur.nl

\author{
Abbreviations \\ FDH Formate dehydrogenase \\ FMDH Formylmethanofuran dehydrogenase \\ $\mathrm{H}_{2}$ ase Hydrogenase \\ ICP-MS Inductively coupled plasma mass spectrometry
}

\section{Introduction}

Syntrophic communities are excellent models to study the metabolic interactions in anaerobic environments (Ishii et al. 2006; Stolyar et al. 2007). Anaerobic syntrophic associations of bacteria and methanogenic archaea operate at the limits of what is thermodynamically possible (Stams et al. 2006; McInerney et al. 2007). Conversion of propionate and other fatty acids is possible only if the productsacetate and, more distinctly, hydrogen or formate-are efficiently reduced to low concentrations by methanogenic archaea. Consequently, interspecies electron transfer plays an important role in the degradation of fatty acids. Syntrophic communities are critical in regulating the flow of carbon and electrons in many anaerobic ecosystems (Schink 2002; Narihiro and Sekiguchi 2007) and are indicators of malfunctioning of the overall biodegradation process in anaerobic bioreactors (Voolapalli and Stuckey 1998).

Many studies focusing on terminal electron transfer have indicated that both formate and hydrogen play a role in interspecies electron transfer carrier (Stams 1994; Schink 1997; de Bok et al. 2004; Batstone et al. 2006). Although the same energy can be obtained by utilization of formate or hydrogen, the different chemical properties of these molecules may affect their relative fluxes across environments or species with diverse physiological characteristics. Low $\mathrm{H}_{2}$ and formate levels are created by methanogenic partners 
that grow on $\mathrm{H}_{2} / \mathrm{CO}_{2}$ and/or formate. Interspecies electron transfer in syntrophic propionate oxidizing consortia has been studied, but it is still unclear whether hydrogen or formate is the preferred electron carrier. Syntrophobacter species oxidize only propionate together with methanogens that utilize both $\mathrm{H}_{2}$ and formate, such as Methanospirillum hungatei and Methanobacterium formicicum, and not with Methanobrevibacter strains that only utilize $\mathrm{H}_{2}$ (Dong et al. 1994). Therefore, besides $\mathrm{H}_{2}$, formate is also considered to play a role in syntrophic propionate oxidation (Thiele and Zeikus 1988; Boone et al. 1989; Stams and Dong 1995). The terminal reductases of a model syntrophic consortium consisting of the propionate-converting Syntrophobacter fumaroxidans and the hydrogen- and formate-scavenging M. hungatei were studied in detail (Dong and Stams 1995; de Bok et al. 2002a). Both the microorganisms possess hydrogenases $\left(\mathrm{H}_{2}\right.$ ases $)$ and formate dehydrogenases (FDHs), of which the activities are much higher during syntrophic growth on propionate compared to the respective pure cultures (de Bok et al. 2002a). Two W-containing FDHs were purified and characterized from S. fumaroxidans (de Bok et al. 2003).

Tungsten (W) and molybdenum (Mo) are essential trace elements for the growth of many anaerobic microorganisms (Kletzin and Adams 1996; Vorholt and Thauer 2002; Schwarz et al. 2007; Andreesen and Makdessi 2008). Several redox-enzymes depend on these metals in obligate anaerobes. Examples of enzymes which contain either W or Mo are: formate dehydrogenase (FDH), which is required for energy conservation and carbon assimilation in anaerobic bacteria and archaea (Ljungdahl and Andreesen 1978; Ferry 1999), formylmethanofuran dehydrogenase (FMDH), which reduces $\mathrm{CO}_{2}$ and couples the formyl group to methanofuran in methanogens (Hochheimer et al. 1998), and a variety of aldehyde oxidizing enzymes and acetylene hydratase (Kletzin and Adams 1996). Since the chemical and catalytic properties of these two metals are very similar, some enzymes contain either Mo or $\mathrm{W}$, and some microorganisms even contain analogues of enzymes containing either $\mathrm{W}$ or Mo, depending on the growth conditions (Bertram et al. 1994; Hochheimer et al. 1998; Stewart et al. 2000; Brondino et al. 2004). However, most microorganisms just possess one type of enzyme, in which the $\mathrm{W}$ and Mo can not effectively replace each other (Jones and Stadtman 1977; Van Bruggen et al. 1986; Zindel et al. 1988; Afshar et al. 1998), and in some cases, they even have antagonistic effects (Ljungdahl and Andreesen 1978; Zellner and Winter 1987; May et al. 1988).

We were interested in the effect of $\mathrm{W}$ and Mo on the growth of $S$. fumaroxidans in syntrophic association with methanogens. In pure culture, $S$. fumaroxidans oxidizes propionate via the methyl-malonyl-CoA pathway and disposes of electrons by either fumarate or sulfate reduction
(Plugge et al. 1993; Stams et al. 1993; Harmsen et al. 1998). Without these electron acceptors, S. fumaroxidans reduces protons to $\mathrm{H}_{2}$ and bicarbonate to formate by means of $\mathrm{H}_{2}$ ase and FDH (Stams and Dong 1995). $\mathrm{H}_{2}$ and formate formation coupled to propionate oxidation are endergonic under standard conditions. Therefore, the levels of $\mathrm{H}_{2}$ and/ or formate have to be kept low in order to make the conversion energetically feasible (Stams 1994; Schink 1997).

In present study, we further examined interspecies electron transfer via hydrogen and formate during anaerobic oxidation of propionate by investigating the importance of $\mathrm{W}$ and Mo on the growth of S. fumaroxidans and M. hungate $i$, and determined the FDH and $\mathrm{H}_{2}$ ase levels in cell extracts. We hypothesized that when interspecies formate transfer is essential, syntrophic growth of the Syntrophobacter-Methanospirillum coculture should be affected by a shortage of tungsten and/or molybdenum. Such experiments have never been described to investigate interspecies electron transfer.

\section{Materials and methods}

\section{Growth conditions}

Syntrophobacter fumaroxidans (DSM10017) and Methanospirillum hungatei (DSM864) were routinely cultured at $37^{\circ} \mathrm{C}$ in $120-\mathrm{ml}$ serum flasks with $50 \mathrm{ml}$ of medium and a gas phase of $172 \mathrm{kPa} \mathrm{N} / \mathrm{CO}_{2}$ or $\mathrm{H}_{2} / \mathrm{CO}_{2}(80: 20$, vol/vol) as described previously (Stams et al. 1993). W and Mo were added as sodium tungstate and sodium molybdate. To study the effect of Mo and W, four different media with different $\mathrm{W}$ and Mo concentrations were used. Medium I contained no $\mathrm{W}$ and Mo; Medium II contained no $\mathrm{W}$ and $0.1 \mu \mathrm{M}$ molybdate; Medium III contained $0.1 \mu \mathrm{M}$ tungstate and no Mo; Medium IV contained $0.1 \mu \mathrm{M}$ tungstate and $0.1 \mu \mathrm{M}$ molybdate.

Propionate, fumarate and formate (all sodium salts) were added from the $1 \mathrm{M}$ sterile stock solutions as indicated in the text. For the pure culture experiments $S$. fumaroxidans was grown on $20 \mathrm{mM}$ propionate plus $60 \mathrm{mM}$ fumarate. $S$. fumaroxidans was adapted to each growth condition by subculturing in fresh medium for six transfers.

The composition of the media used to assess the effects of Mo and $\mathrm{W}$ on $M$. hungatei was similar as described above for $S$. fumaroxidans, except that acetate $(2 \mathrm{mM})$ was added as extra source of carbon, and these cultures were shaken at $150 \mathrm{rpm}$ to prevent gas diffusion limitation. The cultures of $M$. hungate $i$ were adapted to growth on either $\mathrm{H}_{2} / \mathrm{CO}_{2}(172 \mathrm{kPa} ; 80: 20$, vol/vol) or formate $(120 \mathrm{mM})$ as substrate for at least three subsequent transfers in the corresponding media. Growth was followed by measuring methane formation. 
Co-cultures of $S$. fumaroxidans and $M$. hungatei were cultivated on propionate $(30 \mathrm{mM})$ in the above mentioned media with or without Mo and W. The co-cultures were adapted to growth on propionate for at least three subsequent transfers in the corresponding media. Growth was followed by measuring methane formation.

To minimize metal contamination, glassware was soaked in a $4 \mathrm{M}$ nitric acid solution for 3 days; new butylrubber stoppers were soaked overnight and washed thoroughly with nano-pure water before use. All solutions and media were prepared with extra-pure water. All chemicals and vitamins were of the highest grade purity.

\section{Cell separation and fractionation}

Cells were harvested in the late exponential phase by centrifugation under anoxic conditions as described previously (de Bok et al. 2002b). Syntrophically grown cells of $S$. fumaroxidans and M. hungatei were separated by Percoll gradient centrifugation as described before (de Bok et al. 2002b). Separated cells were washed and suspended in $50 \mathrm{mM}$ Tris- $\mathrm{HCl}, \mathrm{pH} 8.0$ plus $100 \mu \mathrm{M}$ sodium dithionite. Cell-free extract was obtained by sonication and cell debris was removed by centrifugation at $16,000 \times g$ (de Bok et al. 2002b).

\section{Enzyme activities}

Activity of formate dehydrogenase and hydrogenase was routinely measured at $37^{\circ} \mathrm{C}$ in $\mathrm{N}_{2}$-flushed or $\mathrm{H}_{2}$-flushed cuvettes containing $1 \mathrm{ml}$ of $50 \mathrm{mM}$ Tris- $\mathrm{HCl}(\mathrm{pH}$ 8) and closed with butyl-rubber stoppers. Formate and $\mathrm{H}_{2}$ oxidation rates were recorded at $578 \mathrm{~nm}$ with $1 \mathrm{mM}$ benzyl viologen $\left(\mathrm{BV}^{+}, \varepsilon=8.65 \mathrm{mM}^{-1} \mathrm{~cm}^{-1}\right)$ as electron acceptor.

One unit of enzyme is defined as the amount of enzyme catalyzing the conversion of $1 \mu \mathrm{mol}$ of substrate per min. The final concentrations of benzyl viologen and formate were 1 and $10 \mathrm{mM}$, respectively, while $\sim 1$ bar of $\mathrm{H}_{2}$ was added into the headspace to measure $\mathrm{H}_{2}$ oxidation rates. Protein was determined according to Bradford with bovine serum albumin as a standard (Bradford 1976).

\section{Analytical methods}

Growth was followed in time by determining the optical density at $600 \mathrm{~nm}$ or by quantifying the methane production. It was assumed that methane formation is linearly correlated to growth. $\mathrm{CH}_{4}$ and $\mathrm{H}_{2}$ were determined on a Chrompack 9001 gas chromatograph (Chrompack Varian International, Bergen op Zoom, the Netherlands) equipped with a PlotFused silica column $(30 \mathrm{~m}$ by $0.53 \mathrm{~mm}$ ) packed with Molsieve 5A $(\mathrm{DF}=15 \mu \mathrm{m})$. The temperature of the column, injection port and thermal conductivity detector were 50, 60 and $130^{\circ} \mathrm{C}$, respectively. Argon was used as carrier gas $(20 \mathrm{ml} / \mathrm{min})$. Metal content of the medium was measured by inductively coupled plasma mass spectrometry (ICP-MS, Elan 6000, Perkin-Elmer) as described by van Hullebusch et al. (2005).

\section{Results and discussion}

Effects of W and Mo on co-cultures of S. fumaroxidans and $M$. hungatei

The FDH and $\mathrm{H}_{2}$ ase activities in cell-free extracts of syntrophically grown $S$. fumaroxidans and $M$. hungatei cells are presented in Table 1. Maximum methane formation rates in these co-cultures were calculated from the methane production curves (Fig. 1). Analysis of our basal medium without $\mathrm{W}$ and Mo revealed that the residual $\mathrm{W}$ and Mo concentrations were below the detection limit of 55 and $10 \mathrm{pM}$, respectively, indicating that chemical contamination as a source of W or Mo in our basal medium was kept to a minimum.

In syntrophically grown cells of $S$. fumaroxidans and $M$. hungatei the highest FDH levels were detected when $\mathrm{W}$ was present in the medium (medium III and IV). These specific activities were much higher than those in axenically grown cells of $S$. fumaroxidans and $M$. hungatei on formate (Table 1), consistent with findings reported previously (de Bok et al. 2002a). In W-depleted media (medium I and II), FDH levels were significantly lower in both the organisms (Table 1). The highest methane formation rate, $0.59 \mathrm{nmol} \mathrm{min}{ }^{-1} \mathrm{ml}^{-1}$, was measured in medium with both $\mathrm{W}$ and Mo, comparable to the rate measured by Dong and Stams (1995). The rate was more or less the same $\left(0.57 \mathrm{nmol} \mathrm{min}^{-1} \mathrm{ml}^{-1}\right)$ when Mo was omitted, but it decreased to $0.41 \mathrm{nmol} \mathrm{min}{ }^{-1} \mathrm{ml}^{-1}$ in W-depleted medium (medium II). In the medium without $\mathrm{W}$ and Mo the methane formation rate was only $0.13 \mathrm{nmol} \mathrm{min}{ }^{-1} \mathrm{ml}^{-1}$, which is at least due to the inhibition of $M$. hungatei by $\mathrm{W}$ - and Mo-limitation as we demonstrated in our experiments with pure cultures (Table 1). The FDH level in M. hungatei under these conditions (no metals added) was $0.02 \mathrm{U} / \mathrm{mg}$ protein. This is almost negligible compared to its level in medium without any limitations, considering that the efficiency of Percoll separation with these co-cultures is never $100 \%$ (de Bok et al. 2002a). Moreover, this activity could be attributed to residual FDH from $S$. fumaroxidans cells in the Percoll separated $M$. hungatei cells. Therefore it is likely that $S$. fumaroxidans and $M$. hungatei grow syntrophically with $\mathrm{H}_{2}$ as the only interspecies electron carrier, when $\mathrm{W}$ and Mo are depleted in the growth medium. Whether the low growth rate can be attributed to the 
Table $1 \mathrm{CH}_{4}$ formation and growth rates, formate dehydrogenase (FDH) and hydrogenase $\left(\mathrm{H}_{2}\right.$ ase $)$ activities in cell extracts of Percoll separated co-cultures S. fumaroxidans and M. hungatei, of $S$. fumaroxidans (grown on propionate plus fumarate) and $M$. hungatei (grown on formate or $\mathrm{H}_{2} / \mathrm{CO}_{2}$ ) after adaptation to growth in media with or without $\mathrm{W}$ and/or Mo

\begin{tabular}{llcrr}
\hline & Medium & FDH (U/mg) & $\mathrm{H}_{2}$ ase $(\mathrm{U} / \mathrm{mg})$ & Growth rate $^{\mathrm{b}}$ \\
\hline S. fumaroxidans & No Mo, no W & $5.5 \pm 0.4$ & $8.2 \pm 0.1$ & $0.13 \pm 0.02$ \\
Cocultured & Mo, no W & $19.5 \pm 1.1$ & $9.5 \pm 0.7$ & $0.41 \pm 0.03$ \\
& No Mo, W & $102 \pm 3.1$ & $9.9 \pm 0.6$ & $0.57 \pm 0.04$ \\
& Mo, W & $95 \pm 0.9$ & $12.7 \pm 1.5$ & $0.59 \pm 0.02$ \\
M. hungatei & No Mo, no W & $0.02 \pm 0.02$ & $0.6 \pm 0.1$ & $0.13 \pm 0.02$ \\
Cocultured & Mo, no W & $2.2 \pm 0.3$ & $0.8 \pm 0.1$ & $0.41 \pm 0.03$ \\
& No Mo, W & $25.5 \pm 1.2$ & $1.8 \pm 0.2$ & $0.57 \pm 0.04$ \\
S. fumaroxidans & Mo, W & $44 \pm 2.1$ & $3.2 \pm 0.4$ & $0.59 \pm 0.02$ \\
& No Mo, no W & $2.5 \pm 0.1$ & $15.6 \pm 0.4$ & $0.21 \pm 0.03$ \\
& Mo, no W & $5.0 \pm 0.5$ & $9.1 \pm 0.4$ & $0.19 \pm 0.01$ \\
& No Mo, W & $25.3 \pm 0.3$ & $7.4 \pm 0.9$ & $0.22 \pm 0.01$ \\
M. hungatei formate & No Mo, no W & $0.3 \pm 0.1$ & $14.5 \pm 2.1$ & $0.11 \pm 0.02$ \\
& Mo, no W & $1.1 \pm 0.1$ & $9.2 \pm 1.2$ & $0.41 \pm 0.03$ \\
& No Mo, W & $7.3 \pm 0.4$ & $5.6 \pm 0.1$ & $0.33 \pm 0.05$ \\
& Mo, W & $4.8 \pm 0.8$ & $2.5 \pm 0.1$ & $0.41 \pm 0.01$ \\
& No Mo, no W & $0.006 \pm 0.0$ & $0.8 \pm 0.1$ & $0.40 \pm 0.16^{\mathrm{a}}$ \\
& Mo, no W & $3.2 \pm 0.1$ & $1.3 \pm 0.1$ & $0.60 \pm 0.07$ \\
& No Mo, W & $18 \pm 0.4$ & $1.7 \pm 0.2$ & $0.76 \pm 0.06$ \\
& Mo, W & $25 \pm 0.5$ & $1.7 \pm 0.2$ & $0.87 \pm 0.08$ \\
\hline
\end{tabular}

a Growth ceased after a short exponential phase, from which the growth rate was calculated

${ }^{\text {b }} \mathrm{CH}_{4}$ formation rates and growth rates are the mean of triplicate measurements and enzyme activities are duplicate measurements and expressed as per day

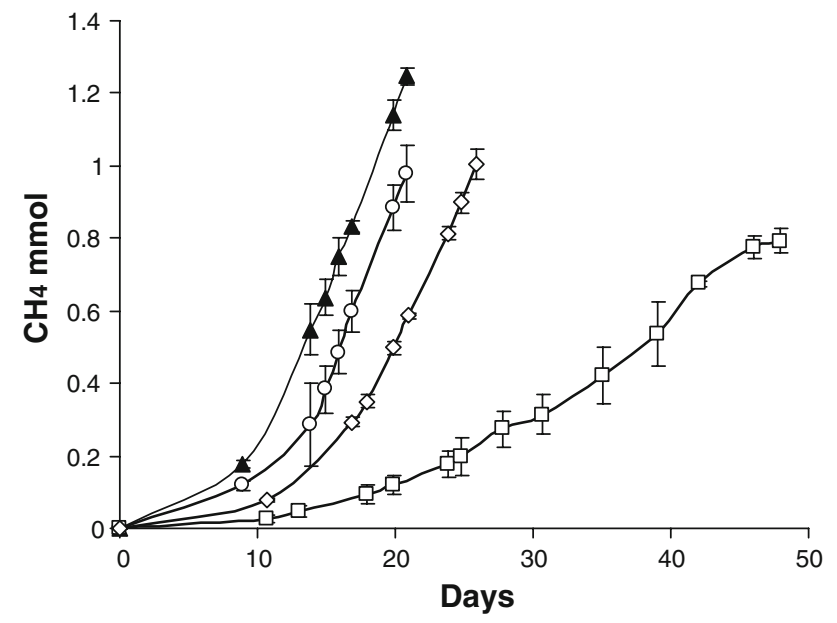

Fig. 1 Syntrophic propionate conversion as determined from methane production by a coculture of $S$. fumaroxidans and $M$. hungatei after three transfers in medium with $\mathrm{W}$ and Mo (triangle), with $\mathrm{W}$ only (circle), with Mo only (diamond) or without $\mathrm{W}$ and Mo (square). Data are the mean of triplicate measurements

absence of interspecies formate transfer alone is not clear, because we do not know how much the M. hungatei FMDH is affected by $\mathrm{W}$ - and Mo-limitation in the co-cultures. In addition, there is also competition for residual $\mathrm{W}$ and $\mathrm{Mo}$ between individual organisms. If $\mathrm{W}$ is required for $\mathrm{CO}_{2}$ reduction in $S$. fumaroxidans for both disposal of electrons (de Bok et al. 2003) and for $\mathrm{CO}_{2}$ fixation for biomass synthesis (Plugge et al. 1993), and the M. hungatei FDH is a Mo-enzyme, the contribution of formate to interspecies electron transfer surely cannot be large. The $\mathrm{CH}_{4}$-formation rate in medium with Mo was only $\sim 1 / 3$ less compared to the rate in medium containing both the metals. Also this finding should be interpreted with caution, since all characterized Mo-containing FDHs, up to now, have less $\mathrm{CO}_{2}$ reductase activity than their $\mathrm{W}$-containing homologues (de Bok et al. 2003). Nevertheless, the data suggest that $\mathrm{H}_{2}$ interspecies transfer is prevailing when Mo is limiting. Further investigation is needed to confirm this. $\mathrm{H}_{2}$ is assumed to be the preferred electron carrier in dense aggregated biomass, whereas formate is more favorable in suspended cultures (Boone et al. 1989; Schmidt and Ahring 1995). Limitation of $\mathrm{W}$ and Mo may also affect the aggregation behavior of microorganisms depending on syntrophic relationships, although such effect has never been reported. Floc formation was observed in syntrophic co-cultures of $S$. fumaroxidans and M. hungatei (de Bok et al. 2002a), and in our experiments we noticed that the formation of aggregates was more evident in cultures without $\mathrm{W}$ than in cultures with $\mathrm{W}$ (results not shown).

Effects of W and Mo on the growth of S. fumaroxidans

Growth rates and maximal optical densities of $S$. fumaroxidans in pure culture were not affected by the absence or presence of $\mathrm{W}$ and/or Mo (Table 1). The average growth rate in each of the different media was around 0.21 per day, which is in the same range as reported previously (Stams et al. 1993). Depletion of W to such level completely inhibited the growth 
of Pyrobaculum aerophilum (Afshar et al. 1998). Therefore, our results suggest that $S$. fumaroxidans does not require $\mathrm{W}$ and Mo addition for growth on propionate and fumarate.

Although the growth rate was not significantly influenced, the Mo and W levels clearly affected the FDH levels in $S$. fumaroxidans that were grown on propionate and fumarate. The lowest FDH level, $2.5 \mathrm{U} / \mathrm{mg}$ of protein, was measured when $S$. fumaroxidans was cultivated in the absence of both W and Mo (Table 1). Addition of either Mo or $\mathrm{W}$ to the medium $(0.1 \mu \mathrm{M}$, medium II and III) resulted in 2- and 10-fold higher FDH levels, respectively. This suggests that both the metals are effectively incorporated in one or more FDHs of $S$. fumaroxidans. Both W and Mo were also effectively incorporated in FDHs of Moorella thermoacetica (Andreesen and Ljungdahl 1973) and Eubacterium acidaminophilum (Granderath 1993). Unlike these two microorganisms, the FDH activity in S. fumaroxidans was substantially lower in cells grown in medium with both $\mathrm{W}$ and Mo, as compared to medium with only W. This was also found for the W- and Mo-containing FDHs of Desulfovibrio alaskensis (Brondino et al. 2004) and dimethyl sulfoxide reductase (DMSO) in Rhodobacter capsulatus (Stewart et al. 2000). In FDH, Mo and W are bound via thiolates to pterin moieties. It is commonly believed, but still not completely resolved that the Molybdenum cofactor (Moco) and the Tungsten cofactor (Wco) are synthesized by a similar and highly conserved pathway (Schwarz et al. 2007). Therefore, when Mo is not present, W is incorporated resulting in a higher specific activity. The two FDHs from $S$. fumaroxidans that were characterized previously appeared to be $\mathrm{W}$-containing enzymes although both the metals were present in the growth medium (de Bok et al. 2003). For S. fumaroxidans FDHs, it would imply that part of the total FDH activity in the cells grown on propionate plus fumarate represents additional FDH(s). Indeed, quantification of different FDHs in S. fumaroxidans suggested that it contains a third FDH (de Bok et al. 2003). Moreover, the genome of $S$. fumaroxidans contains at least two more gene clusters that code for putative formate dehydrogenases (http://img.jgi.doe.gov).

In our study, the FDH levels of cells grown in medium without $\mathrm{W}$ and Mo $(2.5 \mathrm{U} / \mathrm{mg})$ and in medium with only Mo $(5.0 \mathrm{U} / \mathrm{mg})$ could not be addressed to one of the WFDHs that were characterized previously (de Bok et al. 2003). Therefore, the explanation for our observations is that $S$. fumaroxidans possesses one or more constitutively expressed FDH's of which the activity increases when Mo is substituted by $\mathrm{W}$ and substitution of Mo for $\mathrm{W}$ in the two well-studied FDHs does not produce active FDHs. Although FDH activities in $S$. fumaroxidans strongly depend on either $\mathrm{W}$ or Mo, the $\mathrm{H}_{2}$ ase levels in S. fumaroxidans grown on propionate plus fumarate were not significantly affected by the absence of $\mathrm{W}$ and Mo.
Effects of $\mathrm{W}$ and Mo on the growth of M. hungatei

The importance of $\mathrm{W}$ and Mo in methanogenesis has been recognized for a long time. The stimulating effect of both the elements was found to be related to either the FDH levels in methanogens growing on formate, such as M. formicicum and Methanococcus vannielii (Jones and Stadtman 1981; May et al. 1988), or to the FMDH level in methanogens growing on $\mathrm{H}_{2} / \mathrm{CO}_{2}$ such as Methanothermobacter wolfei and Methanothermobacter marburgensis (Bertram et al. 1994; Hochheimer et al. 1998). Growth of M. formicicum and $M$. vannielii was not affected by Mo or $\mathrm{W}$ when $\mathrm{H}_{2} / \mathrm{CO}_{2}$ was the substrate instead of formate, as FDH is not required for autotrophic growth (Jones and Stadtman 1981; May et al. 1988). Similar to these two methanogens, M. hungatei grows on both $\mathrm{H}_{2} / \mathrm{CO}_{2}$ and formate. Therefore, one can expect that growth on formate but not on $\mathrm{H}_{2} / \mathrm{CO}_{2}$ is influenced by $\mathrm{W}$ or Mo. Indeed, the presence of either $\mathrm{W}$ or Mo stimulated growth of $M$. hungatei on formate (Table 1). The highest growth rates ( 0.41 per day) were observed when Mo was present in the medium (medium II and IV). In the absence of Mo but presence of $\mathrm{W}$ the growth rate was slightly lower ( 0.33 per day). When both $\mathrm{W}$ and Mo were absent, the growth rate was only 0.11 per day. The highest FDH level was detected when only $\mathrm{W}$ was present in the medium and it was substantially lower when $\mathrm{W}$ was absent, suggesting the presence of W-FDH(s) in M. hungatei. Apparently, the growth rate of $M$. hungatei was directly related to the FDH activity, as both FDH level and growth rate increased about four times when Mo was added to the medium. But, further increase of FDH level by the addition of $\mathrm{W}$ did not lead to a further increase in growth rate, indicating that something other than FDH is limiting. Analysis of the genome of M.hungatei revealed the presence of three possible FMDHencoding gene clusters (http://img.jgi.doe.gov) that may contain tungsten and could compete for the metals present.

All FDHs from mesophilic methanogens studied so far contain Mo. If we assume that also the M. hungatei FDH(s) contains Mo, our results suggest that $\mathrm{W}$ stimulates the production of Mo-FDH and that W effectively substitutes Mo in $M$. hungatei $\mathrm{FDH}$, resulting in an enzyme with an even higher activity. However, we can not exclude the possibility that $M$. hungatei possesses a W-FDH. Growth of $M$. hungatei on $\mathrm{H}_{2} / \mathrm{CO}_{2}$ was also stimulated by $\mathrm{W}$ and $\mathrm{Mo}$ (Table 1). The highest growth rates of M. hungatei on $\mathrm{H}_{2} /$ $\mathrm{CO}_{2}$ (0.87 and 0.76 per day, respectively) were measured in the cultures grown in the presence of W (Medium III and IV). Growth rates, $\mathrm{FDH}$ and $\mathrm{H}_{2}$ ase levels were substantially lower in the media without W (Medium I and II). When both $\mathrm{W}$ and Mo were absent the culture eventually ceased to grow after a short exponential growth phase.

Our results have implications on the optimization of metal dosage in anaerobic methanogenic bioreactors. It is 
important to maintain balanced communities in the sludge, especially for the $\mathrm{H}_{2}$ and formate consuming methanogens. Maintaining a low partial pressure $\left(\mathrm{H}_{2}\right)$ or concentration (formate) is essential for the degradation of intermediates of anaerobic digestion. Moreover, due to antagonistic effects and competitive uptake of different metals, e.g., cobalt and nickel, tungsten and molybdenum, balanced dosing of all metals is essential to maintain a high biological activity. Overdosing of metals has a toxic effect and may also inhibit the uptake of other essential metals.

Acknowledgments This research was supported by the Research Councils for Earth and Life Sciences (ALW) and Chemical Sciences (CW) with financial aid from the Netherlands Organization for Scientific Research (NWO) and the Technology Foundation (STW), Applied Science Division of NWO.

Open Access This article is distributed under the terms of the Creative Commons Attribution Noncommercial License which permits any noncommercial use, distribution, and reproduction in any medium, provided the original author(s) and source are credited.

\section{References}

Afshar S, Schröder I, Kim C, Monbouquette HG (1998) Effect of tungstate on nitrate reduction by the hyperthermophilic archaeon Pyrobaculum aerophilum. Appl Environ Microbiol 64:3004-3008

Andreesen JR, Ljungdahl LG (1973) Formate dehydrogenase of Clostridium thermoaceticum: incorporation of selenium 75 , and the effects of selenite, molybdate, and tungstate on the enzyme. J Bacteriol 116:867-873

Andreesen JR, Makdessi K (2008) Tungsten, the surprisingly positively acting heavy metal element for prokaryotes. Ann NY Acad Sci 1125:215-229

Batstone DJ, Picioreanu C, van Loosdrecht MCM (2006) Multidimensional modelling to investigate interspecies hydrogen transfer in anaerobic biofilms. Water Res 40:3099-3108

Bertram PA, Schmitz RA, Linder D, Thauer RK (1994) Tungstate can substitute for molybdate in sustaining growth of Methanobacterium thermoautotrophicum. Identification and characterization of a tungsten isoenzyme of formylmethanofuran dehydrogenase. Arch Microbiol 161:220-228

de Bok FAM, Luijten MLGC, Stams AJM (2002a) Biochemical evidence for formate transfer in syntrophic propionate-oxidizing cocultures of Syntrophobacter fumaroxidans and Methanospirillum hungatei. Appl Environ Microbiol 68:4247-4252

de Bok FAM, Roze EHA, Stams AJM (2002b) Hydrogenases and formate dehydrogenases of Syntrophobacter fumaroxidans. Antonie Van Leeuwenhoek 81:283-291

de Bok FAM, Hagedoorn PL, Silva PJ, Hagen WR, Schiltz E, Fritsche K, Stams AJ (2003) Two W-containing formate dehydrogenases $\left(\mathrm{CO}_{2}\right.$-reductases) involved in syntrophic propionate oxidation by Syntrophobacter fumaroxidans. Eur J Biochem 270:2476-2485

de Bok FAM, Plugge CM, Stams AJM (2004) Interspecies electron transfer in methanogenic propionate degrading consortia. Water Res 38:1368-1375

Boone DR, Johnson RL, Liu Y (1989) Diffusion of the interspecies electron carriers $\mathrm{H}_{2}$ and formate in methanogenic ecosystems and its implications in the measurement of $\mathrm{K}_{\mathrm{m}}$ for $\mathrm{H}_{2}$ or formate uptake. Appl Environ Microbiol 55:1735-1741
Bradford MM (1976) A rapid and sensitive method for the quantitation of microgram quantities of protein utilizing the principle of protein-dye binding. Anal Biochem 72:248-254

Brondino CD, Passeggi MC, Caldeira J, Almendra MJ, Feio MJ, Moura JJ, Moura I (2004) Incorporation of either molybdenum or tungsten into formate dehydrogenase from Desulfovibrio alaskensis NCIMB 13491; EPR assignment of the proximal iron-sulfur cluster to the pterin cofactor in formate dehydrogenases from sulfate-reducing bacteria. J Biol Inorg Chem 9:145-151

Dong X, Stams AJM (1995) Evidence for $\mathrm{H}_{2}$ and formate formation during syntrophic butyrate and propionate degradation. Anaerobe $1: 35-39$

Dong X, Plugge CM, Stams AJM (1994) Anaerobic degradation of propionate by a mesophilic acetogenic bacterium in coculture and triculture with different methanogens. Appl Environ Microbiol 60:2834-2838

Ferry JG (1999) Enzymology of one-carbon metabolism in methanogenic pathways. FEMS Microbiol Rev 23:13-38

Granderath K (1993) Charakterisierung der Formiat Dehydrogenase und Aldehyd Dehydrogenase als Wolframhaltige Enzyme von Eubacterium acidaminophilum. $\mathrm{PhD}$ thesis, University of Gottingen, Gottingen

Harmsen HJM, Van Kuijk BLM, Plugge CM, Akkermans ADL, De Vos WM, Stams AJM (1998) Syntrophobacter fumaroxidans sp. nov., a syntrophic propionate-degrading sulfate-reducing bacterium. Int J Syst Bacteriol 48:1383-1387

Hochheimer A, Hedderich R, Thauer RK (1998) The formylmethanofuran dehydrogenase isoenzymes in Methanobacterium wolfei and Methanobacterium thermoautotrophicum: induction of the molybdenum isoenzyme by molybdate and constitutive synthesis of the tungsten isoenzyme. Arch Microbiol 170:389-393

Ishii S, Kosaka T, Hotta Y, Watanabe K (2006) Simulating the contribution of coaggregation to interspecies hydrogen fluxes in syntrophic methanogenic consortia. Appl Environ Microbiol 72:5093-5096

Jones JB, Stadtman TC (1977) Methanococcus vannielii: culture and effects of selenium and tungsten on growth. J Bacteriol 130:14041406

Jones JB, Stadtman TC (1981) Selenium-dependent and seleniumindependent formate dehydrogenases of Methanococcus vannielii. Separation of the two forms and characterization of the purified selenium-independent form. J Biol Chem 256:656-663

Kletzin A, Adams MW (1996) Tungsten in biological systems. FEMS Microbiol Rev 18:5-63

Ljungdahl LG, Andreesen JR (1978) Formate dehydrogenase, a selenium-tungsten enzyme from Clostridium thermoaceticum. Methods Enzymol 53:360-372

May HD, Patel PS, Ferry JG (1988) Effect of molybdenum and tungsten on synthesis and composition of formate dehydrogenase in Methanobacterium formicicum. J Bacteriol 170:3384-3389

McInerney MJ, Rohlin L, Mouttaki H, Kim UM, Krupp RS, Rios-Hernandez L, Sieber J, Struchtemeyer CG, Bhattacharyya A, Campbell JW, Gunsalus RP (2007) The genome of Syntrophus aciditrophicus: life at the thermodynamic limit of microbial growth. Proc Natl Acad Sci 104:7600-7605

Narihiro T, Sekiguchi Y (2007) Microbial communities in anaerobic digestion processes for waste and wastewater treatment: a microbiological update. Curr Opinion Biotechnol 18:273-278

Plugge CM, Dijkema C, Stams AJM (1993) Acetyl-CoA cleavage pathway in a syntrophic propionate oxidizing bacterium growing on fumarate in the absence of methanogens. FEMS Microbiol Lett 110:71-76

Schink B (1997) Energetics of syntrophic cooperation in methanogenic degradation. Microbiol Mol Biol Rev 61:262-280

Schink B (2002) Synergistic interactions in the microbial world. Antonie Van Leeuwenhoek 81:257-261 
Schmidt JE, Ahring BK (1995) Interspecies electron transfer during propionate and butyrate degradation in mesophilic, granular sludge. Appl Environ Microbiol 61:2765-2767

Schwarz G, Hagedoorn P-L, Fischer K (2007) Molybdate and tungstate: uptake, homeostasis, cofactors, and enzymes. In: Nies DH, Silver S (eds) Molecular microbiology of heavy metals. Springer Verlag, Berlin, pp 421-451

Stams AJM (1994) Metabolic interactions between anaerobic bacteria in methanogenic environments. Antonie Van Leeuwenhoek 66:271-294

Stams AJM, Dong X (1995) Role of formate and hydrogen in the degradation of propionate and butyrate by defined suspended cocultures of acetogenic and methanogenic bacteria. Antonie Van Leeuwenhoek 68:281-284

Stams AJM, Van Dijk JB, Dijkema C, Plugge CM (1993) Growth of syntrophic propionate-oxidizing bacteria with fumarate in the absence of methanogenic bacteria. Appl Environ Microbiol 59:1114-1119

Stams AJM, de Bok FAM, Plugge CM, van Eekert MHA, Dolfing J, Schraa G (2006) Exocellular electron transfer in anaerobic microbial communities. Environ Microbiol 8:371-382

Stewart LJ, Bailey S, Bennett B, Charnock JM, Garner CD, McAlpine AS (2000) Dimethylsulfoxide reductase: an enzyme capable of catalysis with either molybdenum or tungsten at the active site. $\mathrm{J}$ Mol Biol 299:593-600

Stolyar S, van Dien S, Linnea Hillesland KL, Pinel N, Lie TJ, Leigh JA, Stahl DA (2007) Metabolic modeling of a mutualistic microbial community. Mol Syst Biol 3:92
Thiele JH, Zeikus JG (1988) Control of interspecies electron flow during anaerobic digestion: significance of formate transfer versus hydrogen transfer during syntrophic methanogenesis in flocs. Appl Environ Microbiol 54:20-29

Van Bruggen JJA, Zwart KB, Hermans GF, van Hove EM, Stum CK, Vogels GD (1986) Isolation and characterization of Methanoplanus endosymbiosus sp. nov., an endosymbiont of the marine sapropelic ciliate Metopus contortus Quennerstedt. Arch Microbiol 144:367-374

Van Hullebusch ED, Peerbolte A, Zandvoort MH, Lens PNL (2005) Sorption of cobalt and nickel on anaerobic granular sludges: isotherms and sequential extraction. Chemosphere 58:493-505

Voolapalli RK, Stuckey DC (1998) Stability enhancement of anaerobic digestion through membrane gas extraction under organic shock loads. J Chem Technol Biotechnol 73:153-161

Vorholt JA, Thauer RK (2002) Molybdenum and tungsten enzymes in C1 metabolism. In: Sigel A, Sigel H (eds) Molybdenum and tungsten. Their roles in biological processes, 'metal ions in biological systems', vol 39. M. Dekker Inc, New York, pp 571-619

Zellner G, Winter J (1987) Growth promoting effect of tungsten on methanogens and incorporation of tungsten-185 into cells. FEMS Microbiol Lett 40:81-87

Zindel U, Freudenberg W, Rieth M, Andreesen JR, Schnell J, Widdel F (1988) Eubacterium acidaminophilum sp. nov., a versatile amino acid-degrading anaerobe producing or utilizing $\mathrm{H}_{2}$ or formate. Description and enzymatic studies. Arch Microbiol 150:254-266 